\title{
Pre-Vascularized Electrospun Graphene Oxide- Gelatin Chamber for Intestinal Wall Defect Repair
}

\author{
Chentao Shen ${ }^{1,2, *}$, Jian Liü ${ }^{3,4, *}$, Qiyi Lu ${ }^{1,2}$, Guihua Wang ${ }^{1,2}$, Zhenxing Wang ${ }^{3,4}$, Lu Liu ${ }^{1,2}$ \\ 'Department of Gastrointestinal Surgery Center, Tongji Hospital, Tongji Medical College, Huazhong University of Science and Technology, Wuhan, \\ 430030, People's Republic of China; ${ }^{2}$ GI Cancer Research Institute, Tongji Hospital, Tongji Medical College, Huazhong University of Science and \\ Technology, Wuhan, 430030, People's Republic of China; ${ }^{3}$ Department of Plastic Surgery, Union Hospital, Tongji Medical College, Huazhong University \\ of Science and Technology, Wuhan, 430022, People's Republic Of China; ${ }^{4}$ Wuhan Clinical Research Center for Superficial Organ Reconstruction, \\ Wuhan, 430022, People's Republic of China
}

*These authors contributed equally to this work

Correspondence: Lu Liu; Zhenxing Wang, Tel +86-I347622682I; +86-I347623 I986, Fax +86-27-83662640; +86-27-85726240,

Email halesan@I63.com; benjamin.wzx@163.com

\begin{abstract}
Purpose: Successful intestinal tissue engineering requires specialized biocompatible scaffolds and a vibrant vascularization microenvironment. A pre-vascularized chamber can provide both in vivo, but there is little report on using it to improve intestinal regeneration. Besides, researchers have found that gelatin is highly biocompatible and graphene oxide (GO) can be used to improve mechanical properties. Thus, applying a pre-vascularized chamber fabricated gelatin and GO into intestinal tissue engineering is worth a try.

Materials and Methods: In this study, an investigation into the physicochemical and mechanical properties as well as biocompatibility of the electrospun graphene oxide-gelatin (GO-Gel) scaffolds were conducted in vitro. Meanwhile, a pre-vascularized GO-Gel (V-GO-Gel) chamber model was built by implanting the scaffold around the mesenteric vessels in rat. After vascularization process, the chamber was used to repair the perforation and then assessed by histology and immunofluorescence analyses.

Results: These porous scaffolds were mechanical improved with GO incorporated into gelatin. Further, the cell adherence, viability and morphology on the scaffolds were maintained. The V-GO-Gel chamber model was successfully built and effective enhanced the repair of the intestinal wall than the other group without recurrence or complications.
\end{abstract}

Conclusion: The V-GO-Gel chamber shows promising therapeutic potential in the repair of intestinal wall defects.

Keywords: graphene oxide, gelatin, pre-vascularization, electrospinning, intestinal wall defect

\section{Introduction}

Tissue regeneration has gained increasing attention in the reconstruction of various body tissues such as the bone and the intestines. ${ }^{1}$ Intestinal tissue regeneration is a complex process with several limitations. ${ }^{2}$ Successful intestinal tissue engineering requires specialized biomaterial scaffolds to support cell adhesion and generation of the extracellular matrix. ${ }^{3}$ Meanwhile, a vascular network, which supply nutrient, oxygen and remove waste, is also vital. ${ }^{4-6}$

In this regard, the vascularized chamber, such as arteriovenous loop (AVL) chamber and flow-through chamber, can support the survival and proliferation of newly-grown tissue. ${ }^{7}$ The vascularized chamber serves as an in vivo bioreactor to provide an isolated and vascularized microenvironment, thus stimulating tissue regeneration. ${ }^{8}$ Various studies have demonstrated different model to enhance intestinal defect repair. ${ }^{9}$ However, only a few studies have attempted to apply the vascularized chamber model in intestinal regeneration or construct tissue engineering chamber with biomaterial.

Natural biomaterials such as gelatin $(\mathrm{Gel})$, collagen, or chitosan, and synthetic biomaterials such as polycaprolactone (PCL), and hydroxyapatite (HA), can be manipulated to simulate the extracellular matrix (ECM). ${ }^{10,11} \mathrm{Gelatin}$ is a natural biomaterial widely used in tissue regeneration. It is a derivative of collagen, acquired by denaturing the triple-helix structure. ${ }^{12}$ Although various studies have revealed that the electrospun gelatin fibers had similar fibers average diameter, mechanical properties and ultimate elongations with collagen fibers. ${ }^{13}$ Gelatin exhibited a higher tensile modulus than 


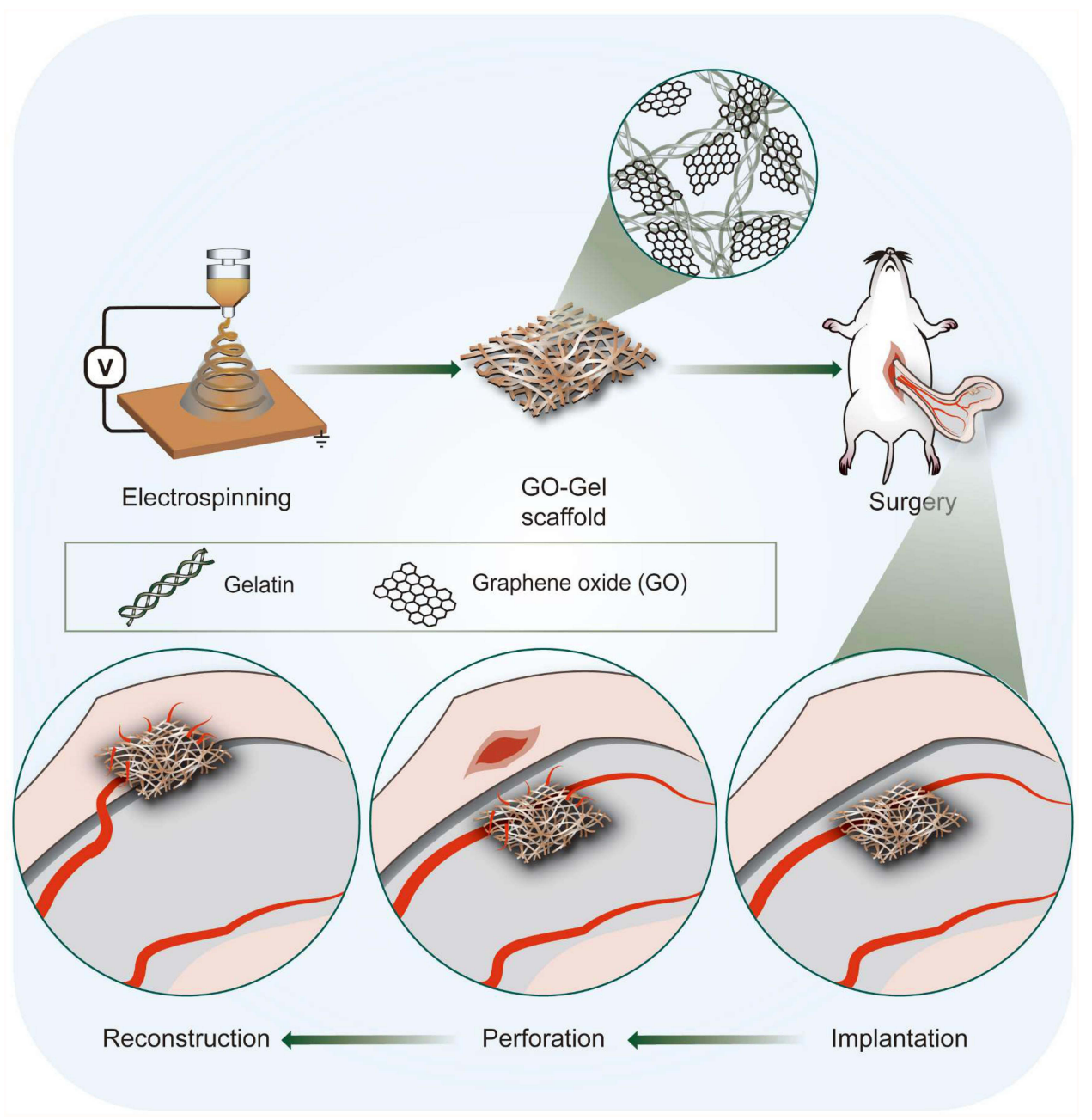

Figure I A schematic diagram of the experimental procedure.

collagen fibers. Also, compared with collagen, gelatin exhibits excellent biodegradability, non-antigenicity, and cost efficiency. ${ }^{13,14}$ However, gelatin has poor mechanical properties. This drawback can be modified using various agents to improve the mechanical properties. ${ }^{15}$

Graphene oxide (GO) is a modified form presenting oxygen-containing functional groups of graphene, a crystalline form of carbon. ${ }^{16}$ With excellent inherent properties, it was recently reported on increasing tensile strength and Young's modulus of biomaterials. ${ }^{17}$ In addition, GO is highly biocompatible with living cells due to the hydrophilic functional groups on the surface. Besides, GO-Gelatin (GO-Gel) scaffold can be fabricated by electrospinning technology, which permits the production of ECM-like three-dimensional structures with nanoscale fibers. ${ }^{18}$ Thus, GO-Gel scaffold is a promising biomaterial to construct tissue engineering chamber.

In this study, we hypothesized that a pre-vascularized GO-Gel (V-GO-Gel) chamber could be optimized to repair intestinal wall defects. Therefore, a nanofibrous GO-Gel scaffold was constructed by electrospinning technology, which permits the production of ECM-like three-dimensional structures with nanoscale fibers. The mechanical and biological properties of the materials were characterized to determine optimal scaffold properties. The V-GO-Gel chamber was built by implanting the scaffold around the mesenteric vessels to ensure vascularization. Then it was harvested and used to repair the wounded intestine. Histology and immunofluorescence assays were used to assess tissue regeneration. Figure 1 is a schematic representation of the experimental design. 


\section{Materials and Methods \\ Fabrication of GO-Gel Scaffolds}

Gelatin (250g Bloom, type B) was purchased from Macklin Biochemical Co. (Shanghai, China). Hexafluoroisopropanol (HFIP), N-(3-Dimethylaminopropyl)-N'-ethylcarbodiimide hydrochloride (EDC), and N-Hydroxysuccinimide (NHS) were purchased from Aladdin Reagent Co. (Shanghai, China). GO was prepared using the Hummer's Method as previously described. ${ }^{19}$ Gelatin was dissolved in HFIP under continuous magnetic stirring for 24 hours to obtain a solution with a gelatin concentration of $50 \% \mathrm{w} / \mathrm{v}$. On the other hand, GO was dissolved in deionized water using ultrasonication in an ice bath for 4 hours to obtain homogeneous GO dispersion with GO concentrations of $0,0.5,1$, and $1.5 \%$ w/v. After that, different concentrations of the GO dispersion were distributed in Gel/HFIP solution using ultra-sonication in an ice bath to obtain homogeneous GO-Gel solution. The electrospinning precursor solution was prepared by mixing gelatin $(25 \% \mathrm{w} / \mathrm{v})$ with $0,0.25,0.5$ and $0.75 \% \mathrm{w} / \mathrm{v}$ GO.

The electrospinning process was improved from the previous studies. ${ }^{20,21}$ The precursor solution for the electrospinning process was filled in a 5-mL syringe attached to a 19-gauge needle. Electrospinning was carried out for 10 hours to generate nanofiber mats using the following parameters: supplied voltage, $18 \mathrm{kV}$; needle tip to collector distance, $20 \mathrm{~cm}$; flow rate, $0.5 \mathrm{~mL} / \mathrm{h}$. Then, the nanofiber scaffolds (Gel, $0.25 \% \mathrm{GO}-\mathrm{Gel}, 0.50 \% \mathrm{GO}-\mathrm{Gel}, 0.75 \% \mathrm{GO}-\mathrm{Gel}$ ) were crosslinked by immersing in a $95 \%$ ethanol solution containing 5\% w/v EDC and 2\% w/v NHS for 24 hours. After a thorough wash in distilled water, the materials were dried overnight in a vacuum oven to remove residual solvents.

\section{Physiochemical Characterization of GO-Gel Scaffolds}

Field Emission Scanning Electron Microscope (FESEM, Nova NanoSEM 450, FEI, Netherlands) set at an accelerating voltage of $10 \mathrm{kV}$ was used to observe the microstructure of the scaffolds. The surface of the scaffolds was sputter-coated with platinum prior to the examination. Thermo Scientific iS50R Nicolet ATR-FTIR and Raman (LabRAM, HR800, Horiba JobinYvon, France) spectrometers were used to record the surface characteristics of the scaffolds. FTIR measurements were determined at a resolution of $4 \mathrm{~cm}^{-1}$ in the frequency range $400-5000 \mathrm{~cm}^{-1}$, while the Raman spectrum was determined at a scanning range of $100-4000 \mathrm{~cm}^{-1}$. A diode laser with a wavelength of $532 \mathrm{~nm}$ and power adjusted to $30 \mathrm{~mW}$ was used as the excitation source. Crystalline phases of GO and GO-Gel materials were evaluated by X-ray diffraction (XRD, Empyrean, PANalytical B.V., Netherlands) with $\mathrm{Cu} \mathrm{K \alpha}$ radiation (20 range 5-90). Data were analyzed using Image $\mathrm{J}$ and OriginPro 9 Software.

\section{Mechanical Property Testing}

Mechanical properties of the scaffolds were determined using electropuls instrument (Instron, UK) with a load cell capacity of $100 \mathrm{~N}$ and speeds of $10 \mathrm{~mm} / \mathrm{min}$. Specimens were cut into rectangular strips $\left(25 \times 5 \mathrm{~mm}^{2}\right)$ with an average thickness of $0.1 \mathrm{~mm}$. Three samples were tested against each concentration of the GO-Gel nanofiber scaffolds. The tensile strength was analyzed using GraphPad Prism 8 Software. Further, Young's modulus was calculated using the OriginPro 9 Software.

\section{In vitro Cytocompatibility of the GO-Gel Scaffolds Cell Culture and Seeding on Scaffolds}

Human umbilical vein endothelial cells (HUVEC: ATCC) and IEC-6 cells (BMCR, China) were used to perform the cytological experiments. Briefly, the cells were cultured in an atmosphere containing $5 \% \mathrm{CO}_{2}$ at $37^{\circ} \mathrm{C}$ using complete Dulbecco's Modified Eagle's Medium (DMEM, Thermo Fisher Scientific, United States) supplemented with 10\% fetal bovine serum (FBS, Thermo Fisher Scientific, United States), 1\% penicillin/streptomycin (Thermo Fisher Scientific, United States) and 0.1 unit/mL human insulin. When the cells reached $80 \%$ confluency, they were washed with phosphate-buffered saline and trypsinized with $0.25 \%$ Trypsin-EDTA for passaging and seeding. The gelatin and GOGel scaffolds were cut into square shapes (length $5 \mathrm{~mm}$ ) and sterilized overnight with ethylene oxide at $37^{\circ} \mathrm{C}$. The sterilized scaffolds were then placed onto a 96-well tissue culture plate and washed thrice with PBS, followed by incubation with complete DMEM for 24 hours. The HUVEC and IEC-6 cells were then seeded into the 96-well culture plates at a density of $3 \times 10^{3}$ and $5 \times 10^{3}$ cells per well, respectively. 


\section{Determination of Cell Viability, Proliferation, Adhesion, and Morphology}

Cell viability and proliferation were determined on the gelatin and GO-Gel scaffolds using the LIVE/DEAD (SigmaAldrich) and MTT assays (Sigma-Aldrich) after one, four, and seven days of incubation per the manufacturer's protocol.

The live and dead cells were stained using Calcein-AM (CAM) and propidium iodide (PI), respectively, and observed using a confocal laser scanning microscope (CLSM, NikonA1Si, Japan.). Green-strained cells were considered living, while red-stained cells were considered dead.

For the MTT assay, $50 \mu \mathrm{L}$ MTT working solution was added to each well and incubated at $37^{\circ} \mathrm{C}$ for 4 hours. After discarding the MTT working solution, $150 \mu \mathrm{L}$ DMSO was added to the wells, and the plate was swayed. Subsequently, $100 \mu \mathrm{L}$ of the supernatant solution was transferred to a new 96-well plate, and the optical density (OD) of the solution was determined using a microplate reader at $490 \mathrm{~nm}$.

Cell adhesion and morphology were determined using FESEM. Briefly, after incubation for two days, the HUVEC and IEC-6 cells on the scaffolds were washed thrice with PBS to remove unattached cells. Subsequently, the cells were fixed with $2.5 \%$ glutaraldehyde solution for 2 hours. Then, the cells were washed again with PBS to remove residual glutaraldehyde. Dehydration was done using increasing concentrations of ethanol (50\% for $60 \mathrm{~min}, 70 \%$ for $30 \mathrm{~min}, 80 \%$ for $20 \mathrm{~min}, 90 \%$ for $15 \mathrm{~min}$, and $100 \%$ for $30 \mathrm{~min}$ ). Finally, the samples were air-dried and coated with platinum in a vacuum for 60 s and observed using FESEM.

\section{In vivo Study on the Repair of the Intestinal Perforation}

\section{Surgical Procedures}

All animal treatments and procedures were conducted according to the ethical guidelines of Tongji Medical College and approved by the Animal Ethical Committee of Tongji Hospital, Huazhong University of Science and Technology (NO. 2019-S069).

Two surgeries were performed in the animal models: Implantation Surgery and Perforation surgery. Male SpragueDawley rats with an initial average weight of 220-250g were allocated into three groups: Gel group ( $\mathrm{n}=5)$, GO-Gel group $(\mathrm{n}=5)$, and $\mathrm{V}-\mathrm{GO}-\mathrm{Gel}$ group $(\mathrm{n}=6)$.

V-GO-Gel group was set as the experimental group, in which the GO-Gel scaffold was first pre-vascularized and then used to repair the intestinal perforation. The rats were anesthetized by intraperitoneal injection of Trichloroacetaldehyde hydrate $(7 \mathrm{~mL} / \mathrm{kg})$. Following shaving and sterilization of the abdominal site, the skin was incised. The mesenteric artery and mesenteric vein that runs from the superior mesenteric artery (SMV) and superior mesenteric vein (SMA), respectively, were identified for the implantation surgery. After that, GO-Gel scaffolds $\left(3.0 \times 1.2 \mathrm{~cm}^{2}\right)$ were implanted around the mesenteric vessels. The scaffolds were folded in half along the long side and fixed using 4-0 Prolene with the mesenteric vessels in between. The skin incisions were closed with 3-0 Prolene sutures using continuous and interrupted suturing.

Perforation surgery was performed four weeks after the implantation surgery to ensure vascularization of the implanted scaffolds. The implanted scaffolds and intestines were exposed along the midline abdominal incision. The implanted scaffolds were harvested from the surrounding tissue preserving the mesenteric vessels as the feeding and draining vessels. A 6-8mm perforation was made along the intestine on the proximal side using a scalpel. After disinfection, the wound was covered with the harvested scaffolds, which were then secured with sutures. The Gel and GO-Gel groups, the control groups, were established using non-vascularized scaffolds. After recovery, all the rats were allowed free access to food and water. The mortalities were recorded in each group. Animals were sacrificed four weeks after the implantation surgery, as well as two and four weeks after the perforation surgery to harvest specimens for further tests.

\section{Histology and Immunofluorescence Staining}

The tissue samples were fixed with $4 \%$ paraformaldehyde, paraffin-embedded, cut into $5 \mu \mathrm{m}$ sections, then dewaxed and dehydrated. The sections were then stained with hematoxylin and eosin (HE) to show granulation and development of new tissues. Masson's Trichrome stain was used to assess collagen deposition. Further, immunofluorescence staining was used to analyze the expression of CD31, $\alpha$-SMA, and CD206 to evaluate neovascularization and inflammatory reaction. Immunofluorescence was evaluated using a confocal laser scanning microscope. 


\section{Statistical Analysis}

Data were expressed as mean \pm standard deviation. In addition, data were analyzed using GraphPad Prism 8 Software. Tukey's $t$-test and one-way ANOVA were used to determine the statistical difference. The level of significance was noted by $*$ and $* *$ referring to a $\mathrm{P}$-value of $<0.05$ and $<0.01$, respectively.

\section{Results}

\section{Morphology of the GO-Gel Scaffolds}

A $25 \% \mathrm{w} / \mathrm{v}$ Gelatin and $0.25 \%, 0.50 \%$, and $0.75 \% \mathrm{w} / \mathrm{v}$ GO concentrations were used to prepare uniform Gel and GO-Gel nanofibrous mats. The macrographs showed that the color of the scaffolds changed from white (Gel scaffold) to light brown (GO-Gel scaffold) following the addition of increasing concentrations of GO (Figure 2A). This phenomenon had been reported in previous study that the intensity of the yellow/brown color increases on increasing GO content of the composites. ${ }^{12}$

The morphology of the formed nanofibrous mats was evaluated using FESEM (Figure 2A). With or without the GO, the nanofibrous mats showed randomly oriented porous 3D structures. The mean diameter of the Gel scaffold was 1685 $\pm 231 \mathrm{~nm}$. However, after adding different concentrations of the GO $(0.25 \%, 0.50 \%$, and $0.75 \%)$, the mean diameter of the scaffold was $1480 \pm 175 \mathrm{~nm}, 1033 \pm 145 \mathrm{~nm}$, and $990 \pm 108 \mathrm{~nm}$, respectively. The decrease of the fiber diameter was probably due to a change in the properties of the electrospinning solution caused by the incorporation of the GO into the nanofibers. Similar result had been reported in literature. ${ }^{12,18}$

\section{Physiochemical Characterization of the GO-Gel Scaffolds}

The characteristic peaks of the amide bond in the Gel and GO-Gel scaffolds were detected using FTIR spectroscopy (Figure 2B). The FTIR spectra showed characteristic peaks of gelatin at $3283 \mathrm{~cm}^{-1}, 1635 \mathrm{~cm}^{-1}, 1531 \mathrm{~cm}^{-1}$, and $1237 \mathrm{~cm}^{-1}$ assigned to amide A, amide I, amide II, and amide III bands, respectively. The GO-Gel scaffolds did not exhibit visible alteration of the FTIR spectra, suggesting that GO blended with the electrospinning solution by physical mixing and not by chemical reaction. ${ }^{22}$

Further, Raman spectroscopy was used to determine the structural characteristics of the GO-Gel nanofibers (Figure 2C). The GO showed a characteristic peak D band at $1323 \mathrm{~cm}^{-1}$ and $\mathrm{G}$ band at $1583 \mathrm{~cm}^{-1}$. Characteristic $\mathrm{D}$ and $\mathrm{G}$ bands were also observed after incorporation of the GO into the gelatin, indicating the presence of GO in the GO-Gel scaffolds. ${ }^{23}$

X-ray diffraction was used to investigate the crystalline phases of the materials (Figure 2D). When $2 \theta$ was $11^{\circ}$, a diffraction peak appeared in GO but disappeared in the GO-Gel scaffolds, indicating that the GO reduced due to the presence of amino groups in gelatin and dispersed into a single layer during electrospinning. ${ }^{4}$

\section{Determination of the Mechanical Properties}

Mechanical properties are a key consideration in determining the applications of the GO-Gel nanofibrous masts as tissue engineering scaffolds. Figure 2E shows the mechanical properties investigated. The tensile strain (Figure 2F) was evaluated at the highest point of the stress-strain curves, while Young's modulus (Figure $2 \mathrm{G}$ ) was determined as the slope of the linear part of the curves. The $0.25 \% \mathrm{GO}-\mathrm{Gel}$ scaffold had the highest tensile strain, while the $0.75 \% \mathrm{GO}-\mathrm{Gel}$ had the lowest tensile strain. However, the difference was not statistically significant. The addition of GO was shown to improve Young's modulus of the GO-Gel scaffolds compared with the gelatin scaffolds. The Young's modulus of Gel, $0.25 \%$ GO-Ge, $0.50 \%$ GO-Gel and $0.75 \%$ GO-Gel group was calculated as follows: $56.04 \pm 0.05 \mathrm{MPa}, 83.74 \pm 4.37 \mathrm{MPa}$, $78.54 \pm 0.89 \mathrm{MPa}, 76.17 \pm 3.96 \mathrm{MPa}$ and $73.69 \pm 0.14 \mathrm{MPa}$, respectively. The Young's modulus of the GO-Gel scaffolds was about $150 \%$ higher than that of the gelatin scaffolds. However, difference between the different GO-Gel scaffolds was not statistically significant. Similar observation of increase in Young's modulus as a result of GO incorporation in nanofiber is reported by Panzavolta et al. ${ }^{12}$ 

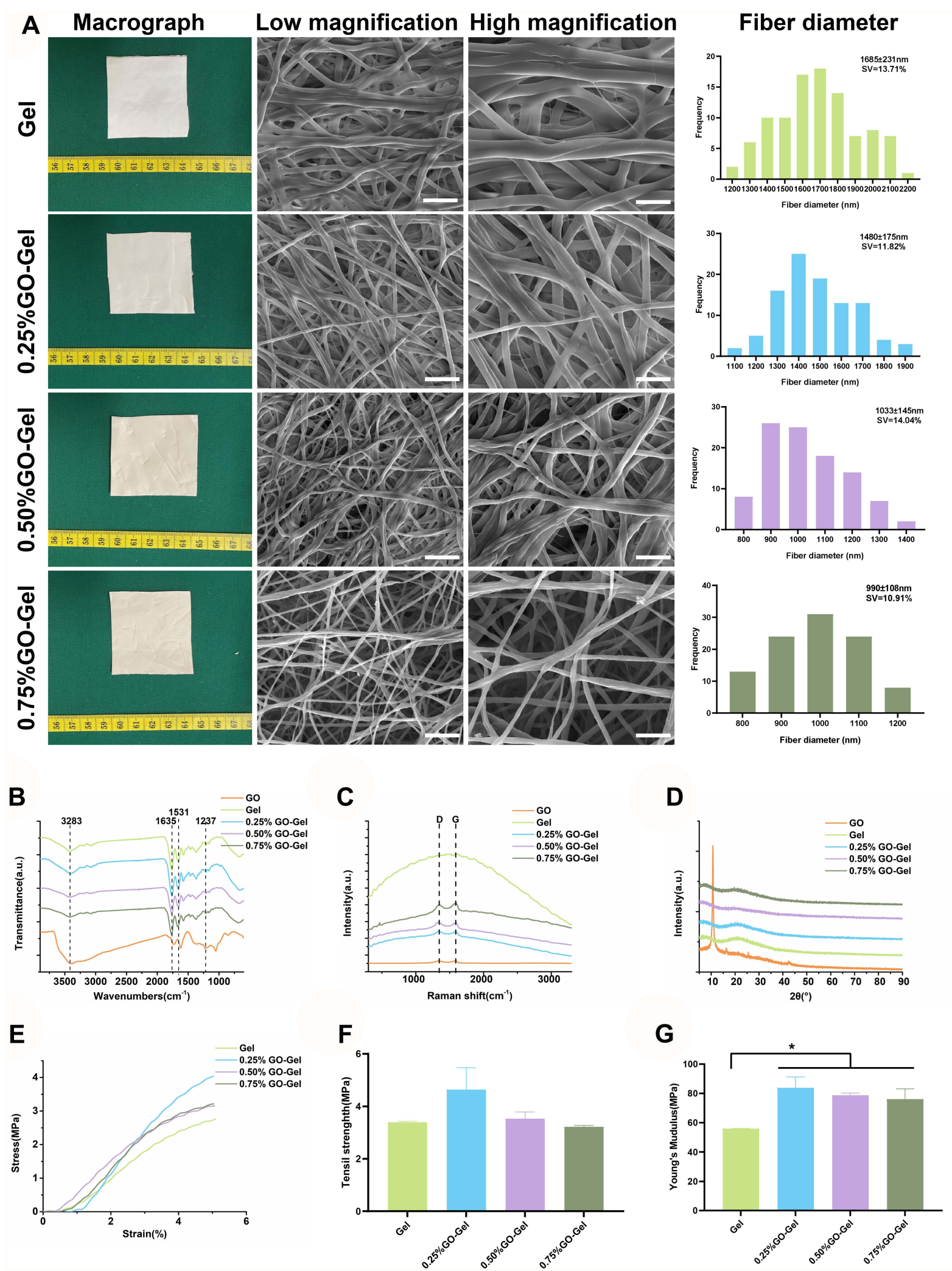

Figure 2 Characterization of the GO-Gel scaffolds. (A) Macrographs, FESEM images, and diameter distribution of the nanofibers (Scale bar: low magnification= $10 \mu \mathrm{m}$, high magnification=5 $\mu \mathrm{m}$ ). (B) FTIR spectroscopy, (C) Raman spectroscopy, (D) XRD pattern, (E) tensile stress-strain curve, (F) tensile strength, and (G) Young's modulus of the scaffolds (mean $\pm S D$, *P-value $<0.05$ ). 


\section{In vitro Cytocompatibility of the GO-Gel Scaffolds}

Cell attachment and proliferation were investigated to evaluate whether the incorporation of GO into gelatin nanofibrous mats satisfied the requirements for tissue engineering. Further, the experiments were carried out to determine the best concentration for use in further experiments. The cells (HUVEC and IEC-6) were cultured on different scaffolds for one, four, and seven days to assess cell viability and proliferation. The HUVEC (Figure 3A) and IEC-6 cells (Figure 3C) showed better cell viability and higher cell proliferation on the other scaffolds in the LIVE/DEAD assay than on the $0.75 \%$ GO-Gel scaffolds. These findings were consistent with the results of the MTT assay of the HUVEC (Figure 3E) and IEC-6 (Figure 3F) cells. Specifically, the $0.25 \%$ GO-Gel scaffold showed the best cell viability and proliferation rates among the different GO-Gel scaffolds, with no statistically significant difference from the pure gelatin scaffolds. The morphology of the HUVEC (Figure 3B) and IEC-6 (Figure 3D) cells attached to the different scaffolds was evaluated after two days of culture using FESEM. The two types of cells showed successful adherence to the scaffolds. Moreover, they showed typical endothelium-like morphology. The cells attached to the scaffolds were colored in blue, while the scaffolds were colored yellow. In summary, the $0.25 \%$ GO-Gel scaffolds showed good cytocompatibility in the in vitro experiments.

\section{The in vivo Study of the Pre-Vascularization Process}

The in vitro studies showed that the mechanical strength of the scaffolds was improved following the incorporation of GO and had good cytocompatibility. Therefore, the scaffolds were used for the intestinal reconstruction model.

The scaffolds were first implanted around the mesenteric vessels to ensure pre-vascularization (Figure 4A). After four weeks, the implanted scaffolds were harvested with the mesenteric vessels preserved as the feeding and draining vessels.

Hematoxylin and eosin (HE) staining, CD31, and $\alpha$-SMA immunofluorescence staining were used to evaluate cell infiltration and formation of blood vessels. In the panorama view of the HE staining for the pre-vascularization of the GO-Gel scaffold, the implanted scaffold was indicated outside the black dotted line, and the central area was within (Figure 4B). In the image of the central area, newly-generated connective tissue could be observed as well as a mount of cell infiltration (Figure 4C). There was increased cell infiltration and neovascularization within the scaffolds (Figure 4D). The CD31 and $\alpha$-SMA immunofluorescence staining showed vascularization of the whole graft area, with a higher density of neovascularization within the scaffold area (Figure 4E-G).

\section{In vivo Study of the Repair Process}

After the pre-vascularization process, the implanted scaffolds were used to repair the intestinal perforation in the V-GOGel group. Meanwhile, the Gel and GO-Gel scaffolds, which were not subjected to prior pre-vascularization process, were used to repair the wounded intestines in the control groups (Figure 5A). After the perforation surgery, three rats died of acute peritonitis within the first three days in the Gel group due to breakage of the scaffolds, while one rat died in the GO-Gel group due to bowel stenosis. All the other rats survived and were sacrificed two or four weeks after the reconstruction surgery (Figure 5B-G). The perforations were evident in all groups after two weeks. However, the rats in the V-GO-Gel scaffolds showed an almost complete wound closure after four weeks. In contrast, wound closure was incomplete after four weeks in the Gel and GO-Gel groups.

The HE staining (Figure 6A-C) and high magnification images (Figure 6D-I) showed tissue regeneration in the three groups. The V-GO-Gel group showed complete bridging of the intestinal perforations with regenerated mucosa. In contrast, perforations sealed with the Gel or GO-Gel scaffolds exhibited apparent and larger gaps in the mucosa. The Masson's trichrome staining showed collagen deposition in the bridged mucosa of the V-GO-Gel group. However, collagen deposition was not evident in the other groups (Figure 6J-R).

Further, immunofluorescence staining against CD31, $\alpha$-SMA, and CD206 was performed to determine the effect of different treatments on neovascularization and inflammatory response on the experimental site (Figure 7). CD31 and $\alpha$ SMA-positive capillaries were mainly concentrated in the scaffolds in the Gel and GO-Gel groups. However, in the V-GO-Gel group, CD31 and $\alpha$-SMA-positive capillaries were concentrated in the bridged mucosa. CD-206 staining showed no differences between the groups (Figure 7A-F). Quantitative analysis showed no differences in the number of neovessels and inflammatory cells between the groups (Figure $7 \mathrm{G}-\mathrm{H}$ ). 

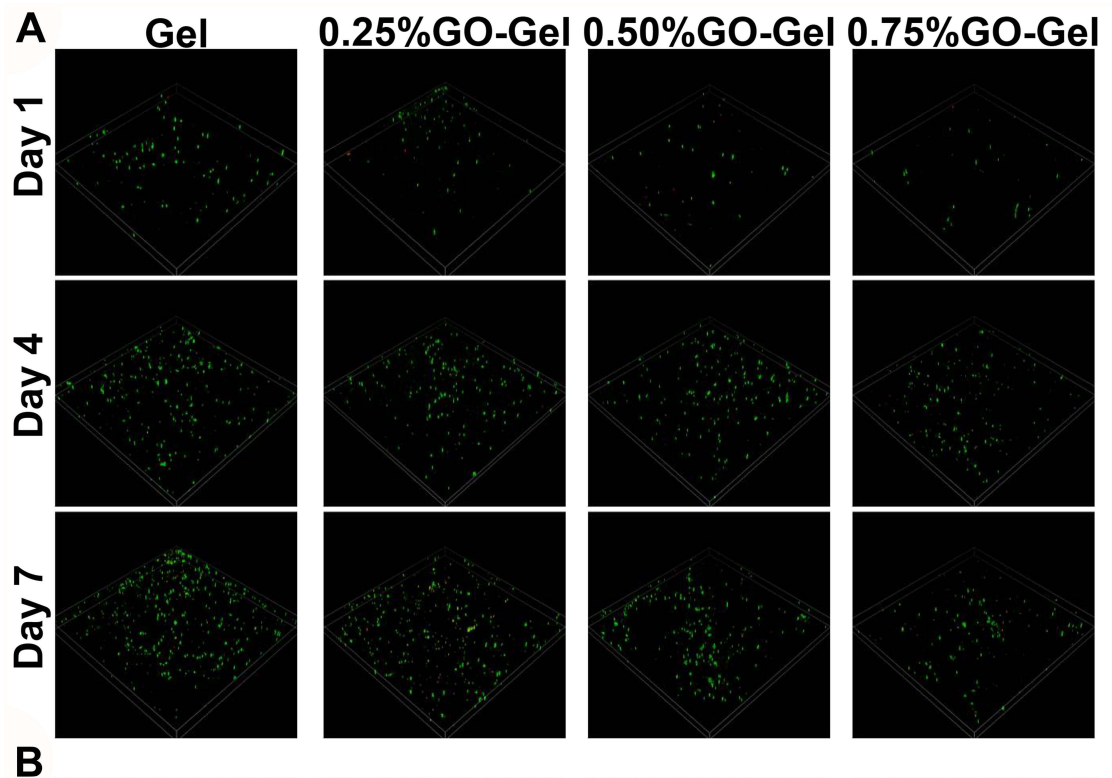

E
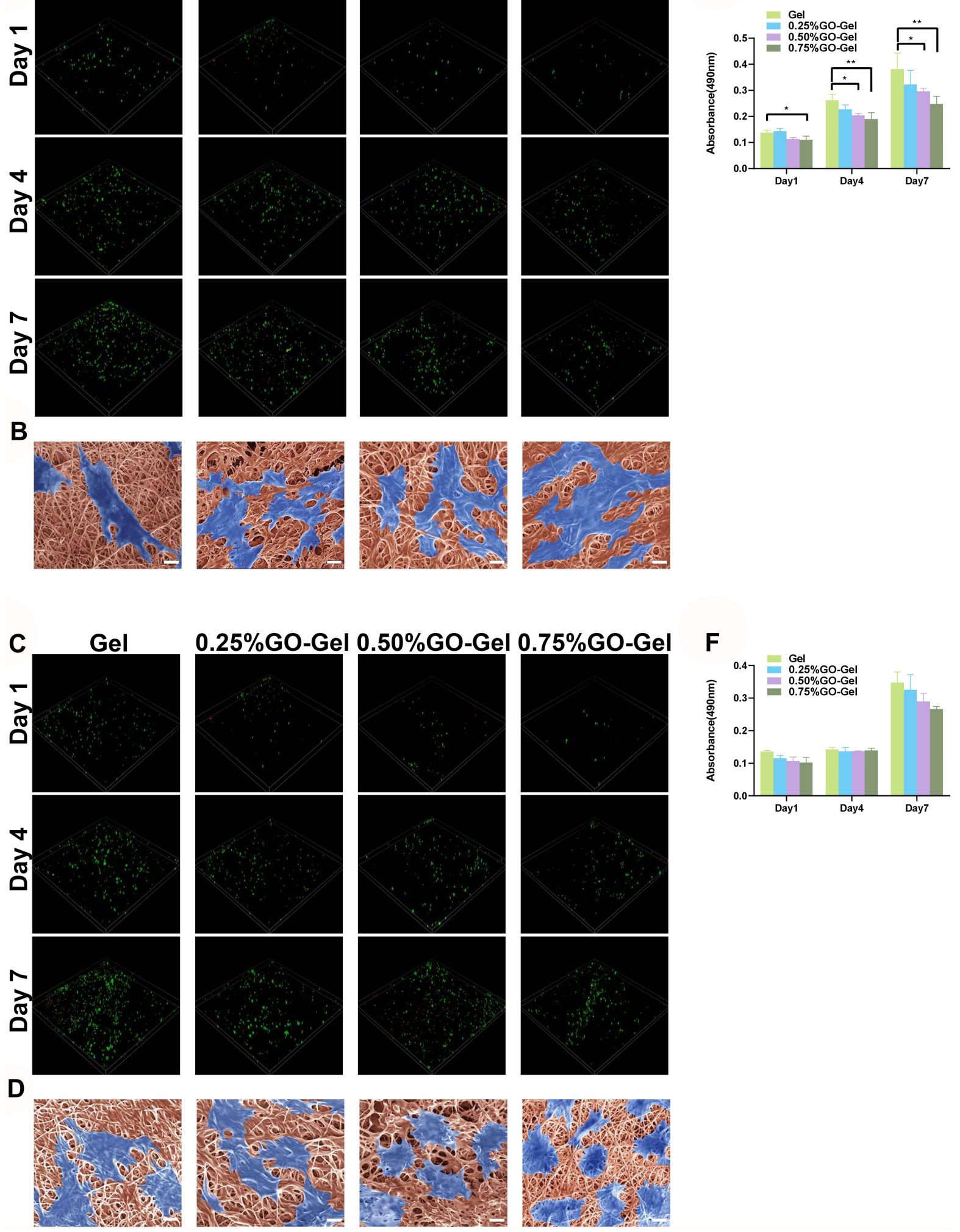

Figure 3 In vitro cytocompatibility of the scaffolds. Confocal fluorescent images showing CAM/PI staining of (A) HUVEC and (C) IEC-6 cells on the scaffolds after culturing for one, four, and seven days. FESEM images showing (B) HUVEC and (D) IEC-6 cells seeded on the scaffolds (scale bar=10 $\mu$ m). Results of the MTT assay performed on (E) HUVEC and (F) IEC- 6 cells seeded on the scaffolds ( $*$ P-value $<0.05$, **P-value $<0.01$ ). 

A 0 weeks
4 weeks
6 weeks
8 weeks
Implantation surgery
·Perforation surgery
-Histological analysis

\section{-Histological analysis $\cdot$ Histological analysis}

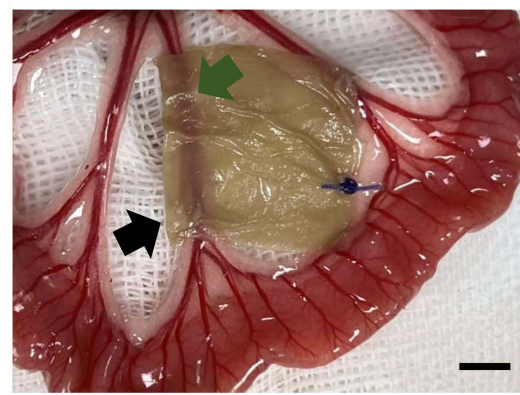

B
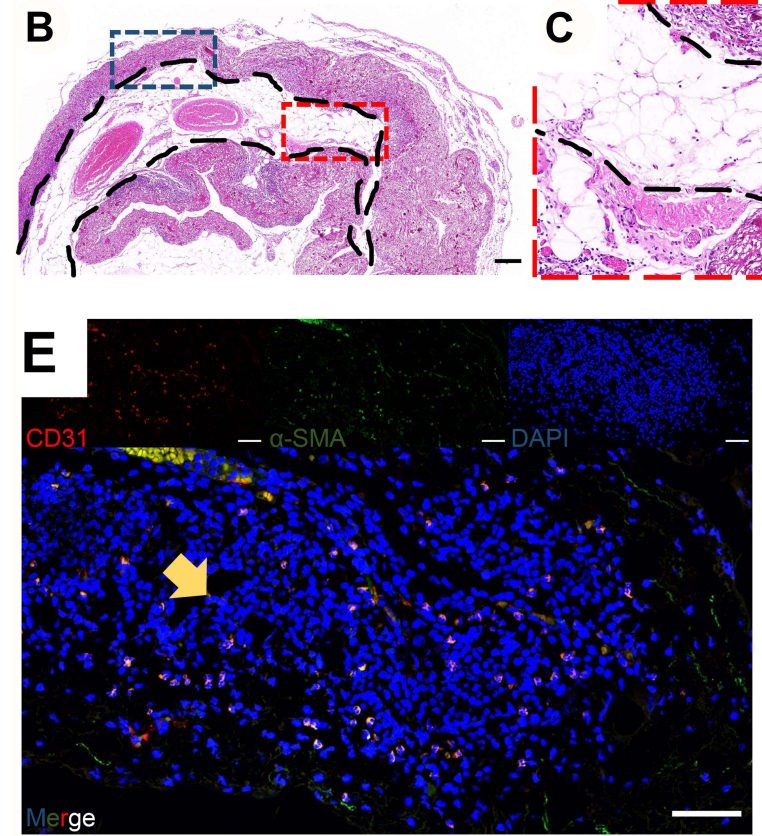

G

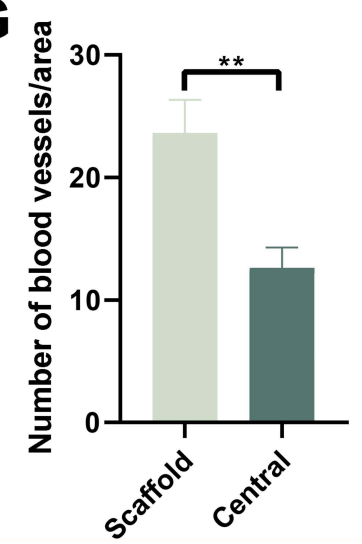

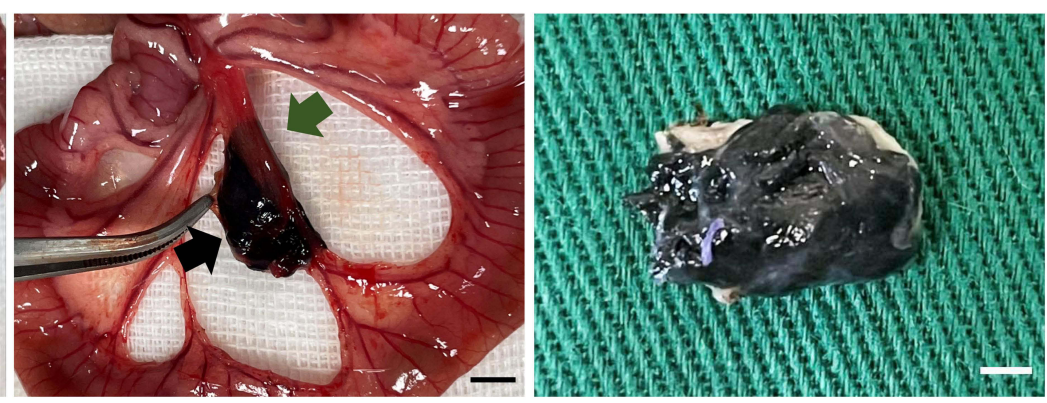
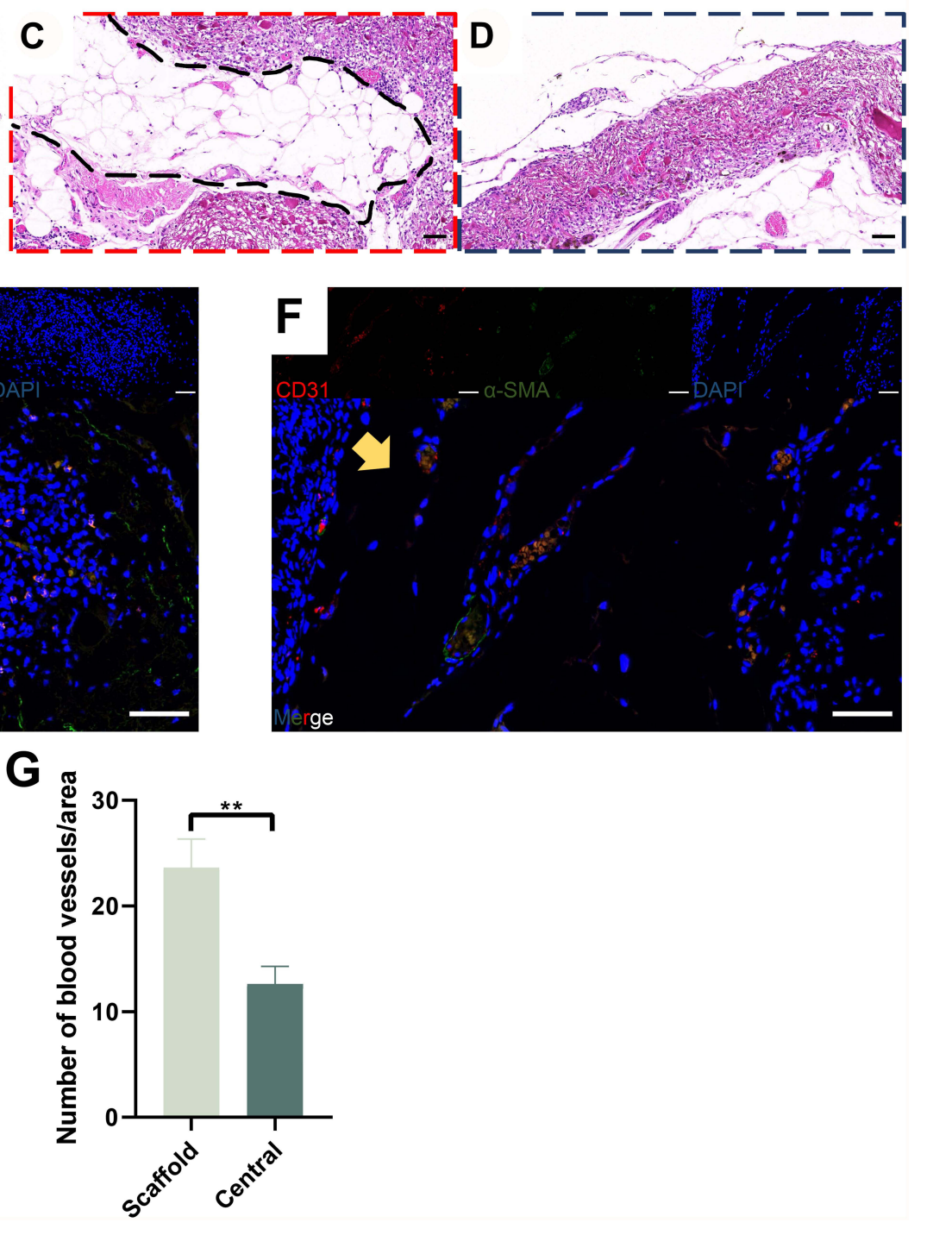

Figure 4 Evaluation of the implantation surgery and pre-vascularization process. (A) Time course of the in vivo experiments and photos of the implantation surgery. The black arrow shows the residual scaffold materials, while the green arrow shows the pre-embedded vessels. (B) Panoramic view of the HE staining of the vascularized scaffolds harvested at week 4. (C) High magnification images of the residual scaffold are marked with blue dotted lines. (D) High magnification images of the central area are marked with red dotted lines. (E) Immunofluorescence images of CD3I, $\alpha$-SMA, DAPI, and merger of the central area. (F) Immunofluorescence images of CD3I, $\alpha$-SMA, DAPI, and merger of the scaffold area. Yellow arrow shows the neovessels. (A scale bar $=5 \mathrm{~mm}$; B scale bar $=200 \mu \mathrm{m}$; $\mathrm{C}-\mathrm{F}$ scale bar=50 $\mu \mathrm{m}$ ). (G) Histogram showing the number of blood vessels/area (mean $\pm \mathrm{SD}$, **P-value $<0.01$ ). 

A
0 weeks
4 weeks
6 weeks
8 weeks
-Implantation surgery
-Perforation surgery
-Histological analysis
-Histological analysis $\cdot$ Histological analysis
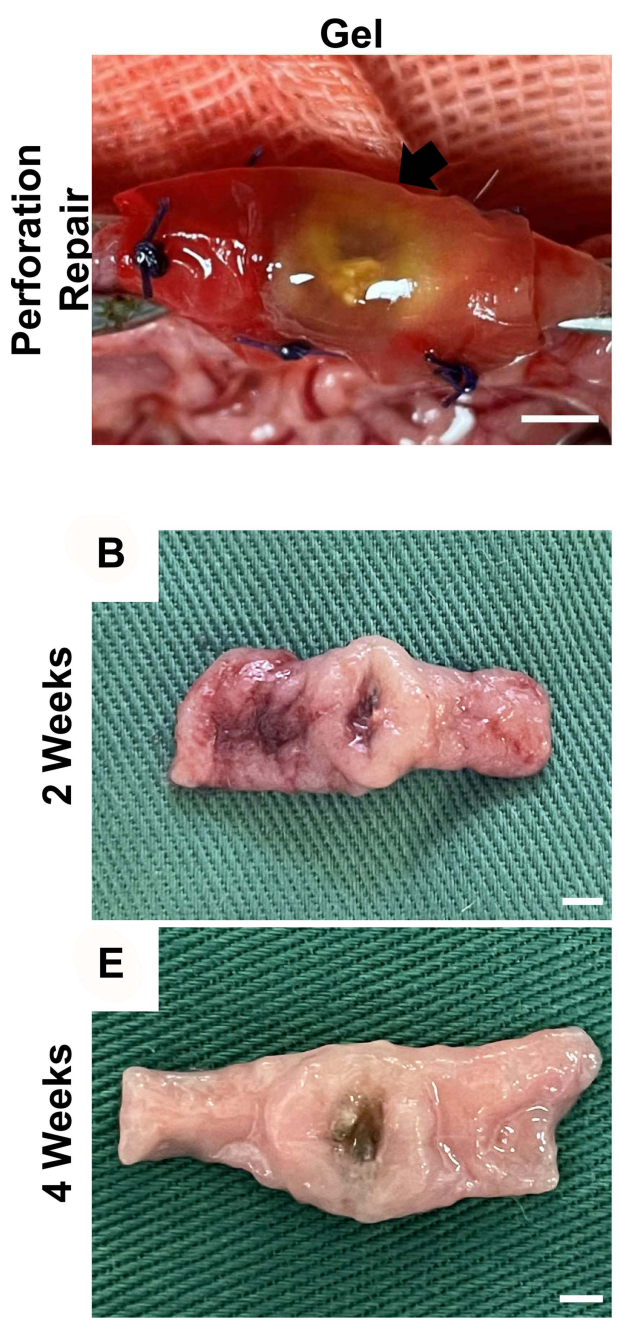
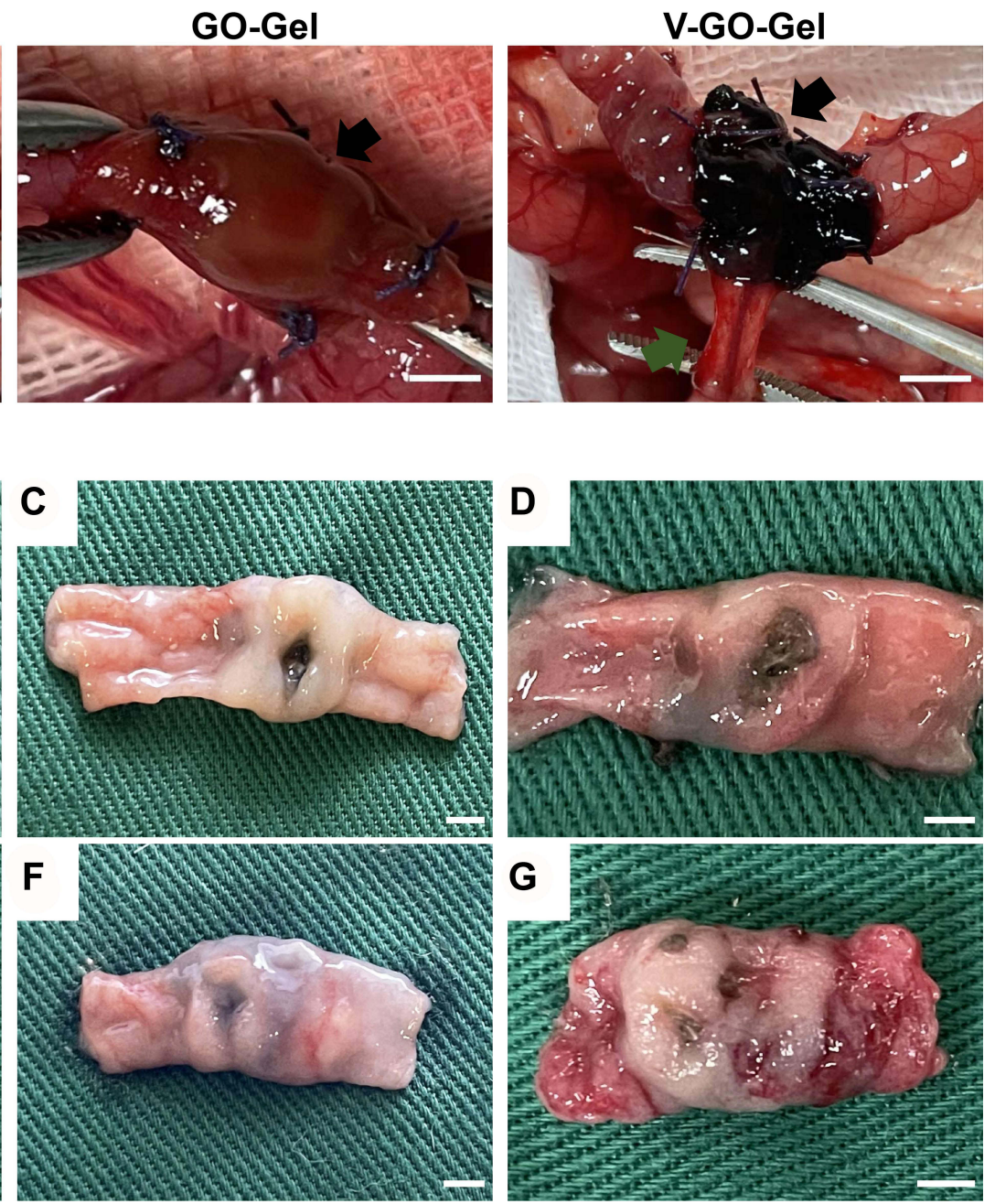

Figure 5 Evaluation of the perforation surgery and repair process. (A) Time course of the in vivo experiments and photos of the perforation surgery. The black arrow shows the perforation and repair area. (B-D) Macroscopic photographs showing the intestinal wounds acquired on week 2 after treatment with different scaffolds. (E-G) Macroscopic photographs showing the intestinal wounds acquired on week 4 after treatment with different scaffolds. (A scale bar=5mm; B-G scale bar=2mm).

Taken together, these results showed that pre-vascularized GO-Gel scaffolds effectively sealed and promoted regeneration of tissues following intestinal perforation, with no severe inflammatory response.

\section{Discussion}

In this study, the pre-vascularized GO-Gel scaffold constructed showed good mechanical properties and adequate vascularization, thus promoting repair of the intestinal perforation in rats. The pre-vascularized GO-Gel group displayed the following advantages over the Gel and GO-Gel groups: (1) enhanced mechanical strength adapted to the intestinal pressure with ability to prevent leakage leading to peritonitis; (2) vascularized microenvironment, thus stimulating regeneration of the intestinal tissue. 

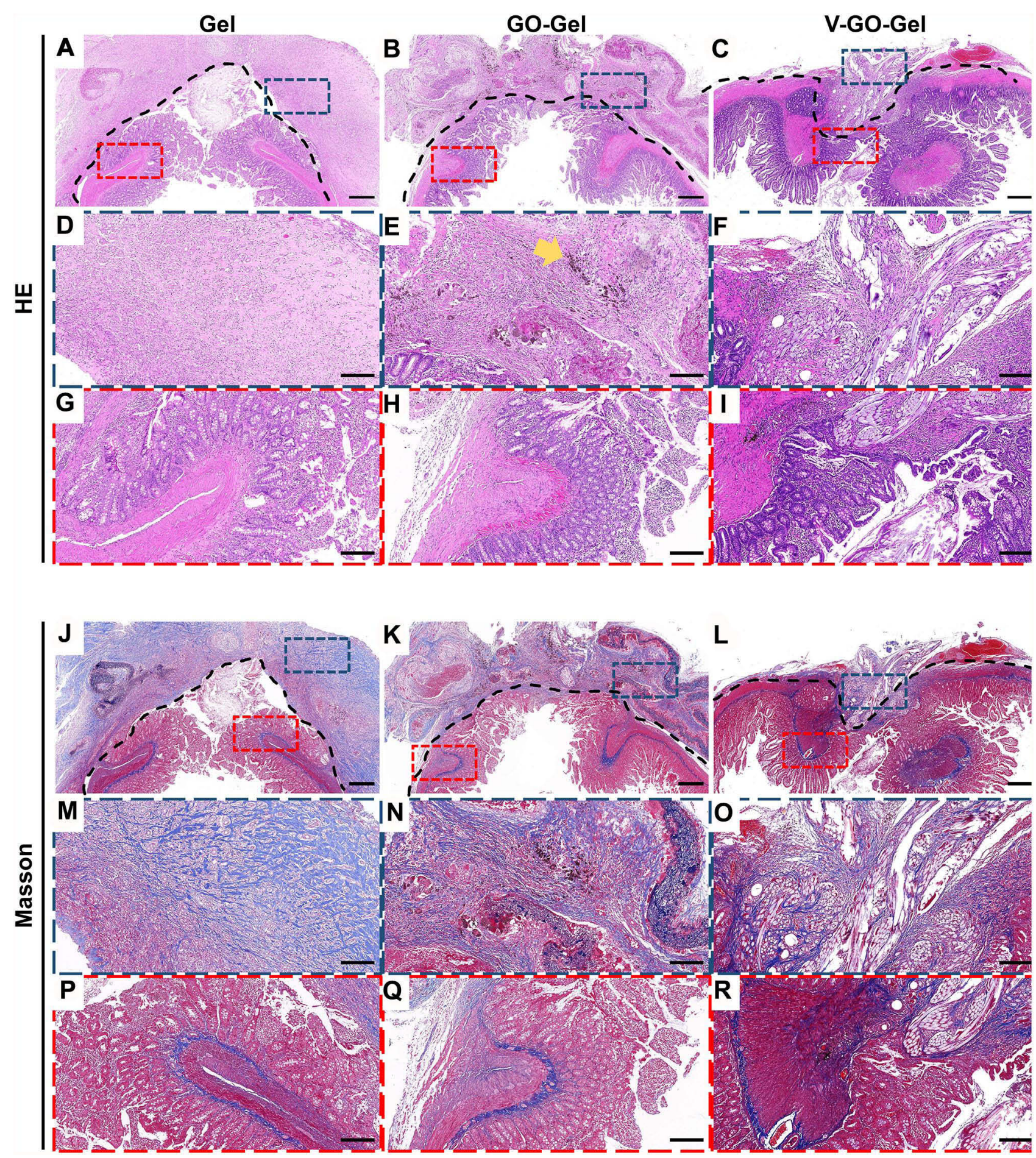

Figure 6 Histological analysis of the vascularized scaffolds 4 weeks after perforation surgery. Panoramic view of the H\&E staining (A-C) and Masson's trichrome staining (JL) of the intestinal wounds acquired on week 4 after treatment with different scaffolds. (scale bar=500um). High magnification images of the HE staining (D-I) and Masson's trichrome staining $(\mathbf{M}-\mathbf{R})$ of intestinal wounds acquired on week 4 after treatment with different scaffolds (scale bar=100um). The yellow arrow indicates the residual graphene oxide sheet. 

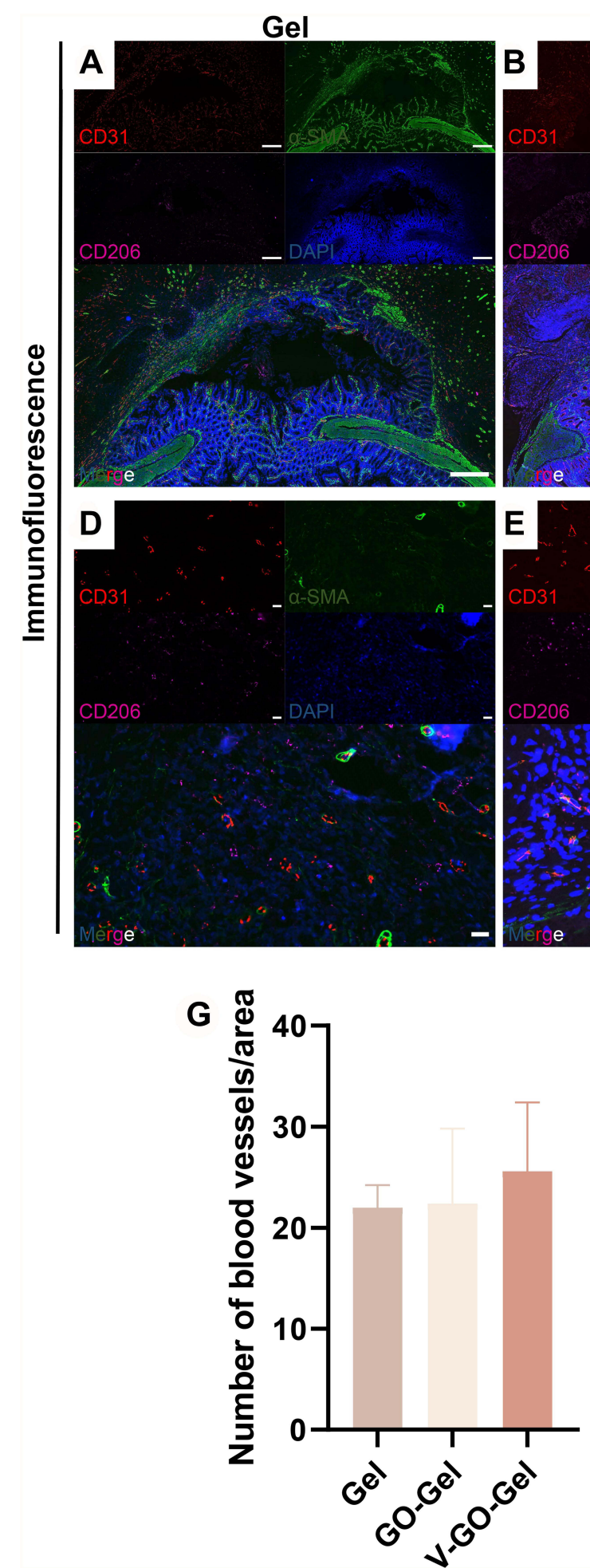

GO-Gel
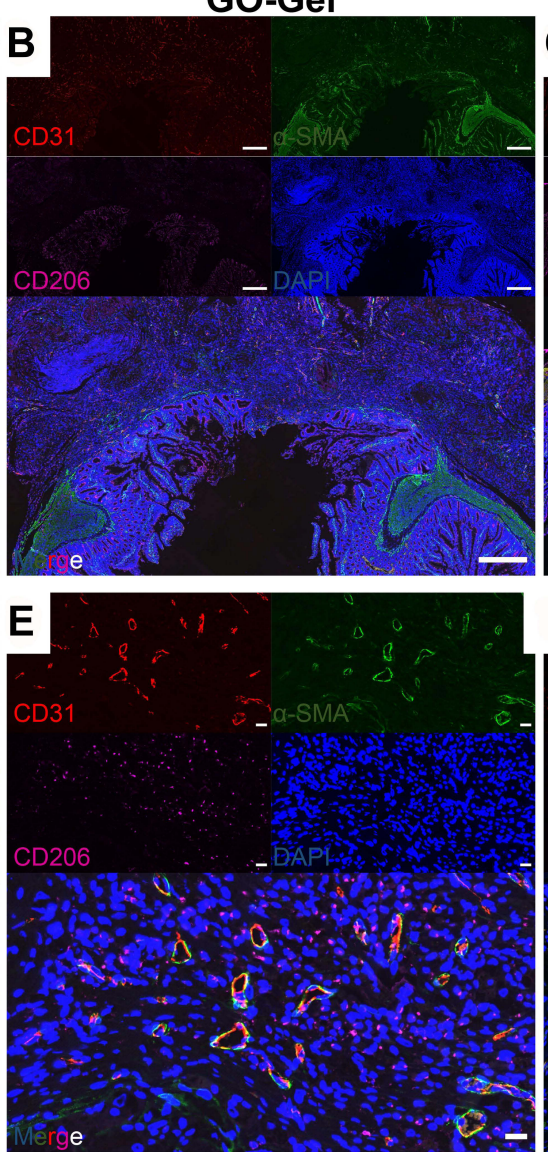

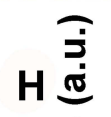

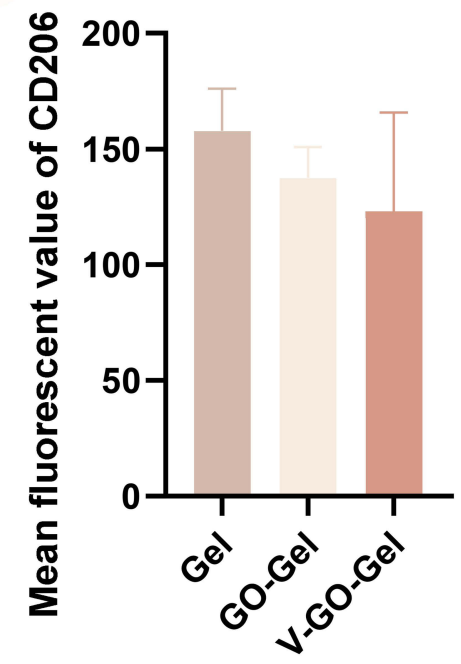

Figure 7 Neovascularization of the intestinal wounds four weeks after perforation surgery. (A-C) Immunofluorescence images of CD3I, $\alpha$-SMA, CD206, DAPI, and merger of intestinal wounds acquired on week 4 after treatment with different scaffolds (scale bar=500 $\mu \mathrm{m}$ ). (D-F) High magnification images of Immunofluorescence images

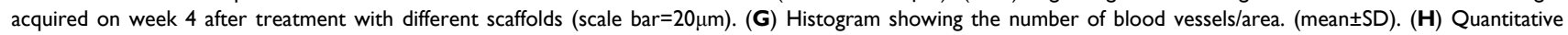
analysis of the CD206 cells (mean $\pm S D$ ). 
An ideal tissue scaffold should have good biostability, which is dictated by the mechanical properties. ${ }^{24}$ Linh et al incorporated biphasic calcium phosphate into gelatin to improve the tensile strength of the electrospun membranes. ${ }^{25}$ Moreover, Satttary et al enhanced Young's modulus and tensile strength of gelatin by adding nHAp. ${ }^{26}$ In this study, the GO-Gel scaffolds were fabricated by electrospinning. The tensile strength and Young's modulus of the mats with low GO concentration were better than that of the pure Gel fibers (Figure 2E-G). Besides, the survival rates in the GO-Gel and V-GO-Gel groups were higher than in the Gel group. This finding suggested improvement in the mechanical strength, thus the anti-leakage effect. The decrease in tensile strength in high GO concentration was due to agglomeration of GO, leading to weak points, hence breakage of the mats. These findings were consistent with results reported by Wan et al and Jalaja et al. ${ }^{18,27}$

Previous studies reported that GO shows biotoxicity. For instance, Wierzbicki et al reported that GO inhibited cell migration and adhesion and showed cell toxicity. ${ }^{28}$ Frontinan-Rubio et al reported that cytotoxicity was associated with increased ROS production. ${ }^{29}$ Thus, the cell proliferation rate was determined using GO-Gel scaffolds fabricated using different concentrations of the GO to determine the optimal concentration of GO within the GO-Gel scaffolds. The results showed that low-concentration GO-Gel scaffold had better cytocompatibility (Figure 3). Moreover, GO can be degraded and eliminated from the body. ${ }^{30}$ Therefore, we determined that $0.25 \%$ GO-Gel scaffold could be used in reconstruction experiments.

Vascularization is the main obstacle in the clinical application of tissue regeneration. Thus, several attempts have been made to develop an ideal vascularization strategy. ${ }^{31}$ The tissue engineering chamber model entails pre-vascularization using pre-embedded vessels to form membrane blebs that lead to vascular sprouts and assembly of neovessels into a robust capillary bed to support the survival of various constructs. Vascularization is mainly achieved using an arteriovenous loop chamber or flow-through chamber models. In this study, a flow-through chamber model was adopted and modified to repair the intestinal perforation (Figures 4 and 6).

The omentum is composed of blood vessels arranged in an arch similar to the intestinal mesentery. It is a commonly used site to develop vascularized pedicles for the reconstruction of wounded intestines. However, the omentum vessels are not suitable for the development of the vascularization model as they are much smaller and denser. ${ }^{32}$ The mesentery is the main source of blood supply to the native intestine. The mesenteric vessels emerge from the "root region", which corresponds to the attachment of the superior mesenteric artery to the aorta. The mesenteric artery then extends radially to the intestinal margin. ${ }^{33}$ Therefore, in this study, mesenteric vessels were selected to be the feeding and draining vessels. While using the chamber to repair the defect, it can restore the local normal anatomy. Furthermore, the prevascularization scaffold model was successfully established using mesenteric vessels. The blood vessels were kept intact during the pre-vascularization process. The GO-Gel scaffold implanted was shown to be highly vascularized (Figure 4). Moreover, the results demonstrated that the pre-vascularization scaffold model showed enhanced intestinal regeneration compared to the non-vascularized groups (Figures 5 and 6). The neovessels were concentrated on the bridge mucosa in the V-GO-Gel group. However, in the other groups, the neovessels were concentrated in the scaffolds (Figure 7). And all these groups did cause critical inflammatory response. This finding may be explained by the fact that the neovessels in the pre-vascularized GO-Gel scaffold supplied nutrients and oxygen, thus promoting regeneration of the intestine in the V-GO-Gel group. However, the non-vascularized group had first to vascularize the scaffolds attached to the perforation.

This study had some limitations. First, the constructed model was simplified compared to the original tissue engineering chamber. Therefore, the regeneration process could not be completely controlled. Therefore, future studies should combine stem cell therapy with biomaterials such as decellularized intestines to improve the model.

In summary, we fabricated a GO-Gel scaffold with enhanced mechanical properties and increased biocompatibility. In addition, a pre-vascularized GO-Gel chamber model was shown to accelerate the process of intestinal reconstruction.

\section{Conclusion}

In conclusion, we successfully repaired the intestinal perforation with the pre-vascularized GO-Gel chamber. Our findings revealed that the scaffolds had high mechanical strength, thus preventing peritonitis and death due to leakage of intestinal contents. Besides, pre-vascularization could enhance the reconstruction of intestinal perforation. 


\section{Acknowledgments}

This study was funded by National Key R\&D Program of China (2019YFA0110500), the National Natural Science Foundation of China (No. 82072198, and 81701922).

\section{Disclosure}

The authors report no conflicts of interest in this work.

\section{References}

1. Yin S, Zhang W, Zhang Z, Jiang X. Recent advances in scaffold design and material for vascularized tissue-engineered bone regeneration. Adv Healthc Mater. 2019;8(10):e1801433. doi:10.1002/adhm.201801433

2. Qi D, Shi W, Black AR, et al. Repair and regeneration of small intestine: a review of current engineering approaches. Biomaterials. 2020;240:119832. doi:10.1016/j.biomaterials.2020.119832

3. Dosh RH, Jordan-Mahy N, Sammon C, Le Maitre CL. Tissue engineering laboratory models of the small intestine. Tissue Eng Part B ReV. 2018;24 (2):98-111.

4. Fang H, Luo C, Liu S, et al. A biocompatible vascularized graphene oxide (GO)-collagen chamber with osteoinductive and anti-fibrosis effects promotes bone regeneration in vivo. Theranostics. 2020;10(6):2759-2772. doi:10.7150/thno.42006

5. Rnjak-Kovacina J, Gerrand YW, Wray LS, et al. Vascular pedicle and microchannels: simple methods toward effective in vivo vascularization of 3D scaffolds. Adv Healthc Mater. 2019;8(24):e1901106. doi:10.1002/adhm.201901106

6. Morrison WA, Marre D, Grinsell D, Batty A, Trost N, O'Connor AJ. Creation of a large adipose tissue construct in humans using a tissue-engineering chamber: a step forward in the clinical application of soft tissue engineering. Ebiomedicine. 2016;6:238-245. doi:10.1016/j. ebiom.2016.03.032

7. Mitchell GM, Morrison WA. In vitro and in vivo approaches for pre-vascularization of 3-dimensional engineered tissues. In: Holnthoner W, Banfi A, Kirkpatrick J, Redl H, editors. Vascularization for Tissue Engineering and Regenerative Medicine. Cham: Springer International Publishing; 2017:1-27.

8. Yap KK, Yeoh GC, Morrison WA, Mitchell GM. The vascularised chamber as an in vivo bioreactor. Trends Biotechnol. 2018;36(10):1011-1024. doi:10.1016/j.tibtech.2018.05.009

9. Sugimoto S, Kobayashi E, Fujii M, et al. An organoid-based organ-repurposing approach to treat short bowel syndrome. Nature. 2021;592 (7852):99-104. doi:10.1038/s41586-021-03247-2

10. Li J, Zhou C, Luo C, et al. N-acetyl cysteine-loaded graphene oxide-collagen hybrid membrane for scarless wound healing. Theranostics. 2019;9 (20):5839-5853. doi:10.7150/thno.34480

11. Wang Q, Feng Y, He M, et al. A hierarchical janus nanofibrous membrane combining direct osteogenesis and osteoimmunomodulatory functions for advanced bone regeneration. Adv Funct Mater. 2020;31(8):2008906. doi:10.1002/adfm.202008906

12. Panzavolta S, Bracci B, Gualandi C, et al. Structural reinforcement and failure analysis in composite nanofibers of graphene oxide and gelatin. Carbon. 2014;78:566-577. doi:10.1016/j.carbon.2014.07.040

13. Heydarkhan-Hagvall S, Schenke-Layland K, Dhanasopon AP, et al. Three-dimensional electrospun ECM-based hybrid scaffolds for cardiovascular tissue engineering. Biomaterials. 2008;29(19):2907-2914. doi:10.1016/j.biomaterials.2008.03.034

14. Zhang S, Huang Y, Yang X, et al. Gelatin nanofibrous membrane fabricated by electrospinning of aqueous gelatin solution for guided tissue regeneration. J Biomed Mater Res A. 2009;90(3):671-679. doi:10.1002/jbm.a.32136

15. Sell SA, McClure MJ, Garg K, Wolfe PS, Bowlin GL. Electrospinning of collagen/biopolymers for regenerative medicine and cardiovascular tissue engineering. Adv Drug Deliv Rev. 2009;61(12):1007-1019. doi:10.1016/j.addr.2009.07.012

16. Shin SR, Li YC, Jang HL, et al. Graphene-based materials for tissue engineering. Adv Drug Deliv Rev. 2016;105:255-274. doi:10.1016/j. addr.2016.03.007

17. Figueroa T, Aguayo C, Fernandez K. Design and characterization of chitosan-graphene oxide nanocomposites for the delivery of proanthocyanidins. Int J Nanomedicine. 2020;15:1229-1238. doi:10.2147/IJN.S240305

18. Jalaja K, Sreehari VS, Kumar PRA, Nirmala RJ. Graphene oxide decorated electrospun gelatin nanofibers: fabrication, properties and applications. Mater Sci Eng C Mater Biol Appl. 2016;64:11-19. doi:10.1016/j.msec.2016.03.036

19. Wang P, Wang X, Tang Q, et al. Functionalized graphene oxide against U251 glioma cells and its molecular mechanism. Mater Sci Eng C Mater Biol Appl. 2020;116:111187. doi:10.1016/j.msec.2020.111187

20. Jafari A, Amirsadeghi A, Hassanajili S, Azarpira N. Bioactive antibacterial bilayer PCL/gelatin nanofibrous scaffold promotes full-thickness wound healing. Int J Pharm. 2020;583:119413. doi:10.1016/j.ijpharm.2020.119413

21. Deng L, Li Y, Zhang A, Zhang H. Nano-hydroxyapatite incorporated gelatin/zein nanofibrous membranes: fabrication, characterization and copper adsorption. Int J Biol Macromol. 2020;154:1478-1489. doi:10.1016/j.ijbiomac.2019.11.029

22. Gao Y, Wang Y, Wang Y, Cui W. Fabrication of gelatin-based electrospun composite fibers for anti-bacterial properties and protein adsorption. Mar Drugs. 2016;14(10):192. doi:10.3390/md14100192

23. Magaz A, Li X, Gough JE, Blaker JJ. Graphene oxide and electroactive reduced graphene oxide-based composite fibrous scaffolds for engineering excitable nerve tissue. Mater Sci Eng C Mater Biol Appl. 2021;119:111632. doi:10.1016/j.msec.2020.111632

24. Ranganathan S, Balagangadharan K, Selvamurugan N. Chitosan and gelatin-based electrospun fibers for bone tissue engineering. Int J Biol Macromol. 2019;133:354-364. doi:10.1016/j.ijbiomac.2019.04.115

25. Linh NT, Lee KH, Lee BT. Functional nanofiber mat of polyvinyl alcohol/gelatin containing nanoparticles of biphasic calcium phosphate for bone regeneration in rat calvaria defects. J Biomed Mater Res A. 2013;101(8):2412-2423. doi:10.1002/jbm.a.34533 
26. Sattary M, Khorasani MT, Rafienia M, Rozve HS. Incorporation of nanohydroxyapatite and vitamin D3 into electrospun PCL/Gelatin scaffolds: the influence on the physical and chemical properties and cell behavior for bone tissue engineering. Polym Adv Technol. 2018;29(1):451-462. doi:10.1002/pat.4134

27. Wan C, Frydrych M, Chen B. Strong and bioactive gelatin-graphene oxide nanocomposites. Soft Matter. 2011;7(13):6159-6166. doi:10.1039/ c1sm05321c

28. Wierzbicki M, Jaworski S, Kutwin M, et al. Diamond, graphite, and graphene oxide nanoparticles decrease migration and invasiveness in glioblastoma cell lines by impairing extracellular adhesion. Int J Nanomedicine. 2017;12:7241-7254. doi:10.2147/IJN.S146193

29. Frontinan-Rubio J, Gomez MV, Martin C, Gonzalez-Dominguez JM, Duran-Prado M, Vazquez E. Differential effects of graphene materials on the metabolism and function of human skin cells. Nanoscale. 2018;10(24):11604-11615. doi:10.1039/C8NR00897C

30. Tonelli FM, Goulart VA, Gomes KN, et al. Graphene-based nanomaterials: biological and Medical applications and toxicity. Nanomedicine. 2015;10(15):2423-2450. doi:10.2217/nnm.15.65

31. Paek J, Park SE, Lu Q, et al. Microphysiological engineering of self-assembled and perfusable microvascular beds for the production of vascularized three-dimensional human microtissues. ACS Nano. 2019;13(7):7627-7643. doi:10.1021/acsnano.9b00686

32. Liu Y, Cromeens BP, Wang Y, et al. Comparison of different in vivo incubation sites to produce tissue-engineered small intestine. Tissue Eng Part A. 2018;24(13-14):1138-1147. doi:10.1089/ten.tea.2017.0313

33. Coffey JC, O'Leary DP. The mesentery: structure, function, and role in disease. Lancet Gastroenterol Hepatol. 2016;1(3):238-247. doi:10.1016/ S2468-1253(16)30026-7

\section{Publish your work in this journal}

The International Journal of Nanomedicine is an international, peer-reviewed journal focusing on the application of nanotechnology in diagnostics, therapeutics, and drug delivery systems throughout the biomedical field. This journal is indexed on PubMed Central, MedLine, CAS, SciSearch ${ }^{\mathbb{R}}$, Current Contents ${ }^{\circledR} /$ Clinical Medicine, Journal Citation Reports/Science Edition, EMBase, Scopus and the Elsevier Bibliographic databases. The manuscript management system is completely online and includes a very quick and fair peer-review system, which is all easy to use. Visit http:// www.dovepress.com/testimonials.php to read real quotes from published authors.

Submit your manuscript here: https://www.dovepress.com/international-journal-of-nanomedicine-journal 